\title{
Implications of short-range spatial variation of soil bulk density for adequate field-sampling protocols: methodology and results from two contrasting soils.
}

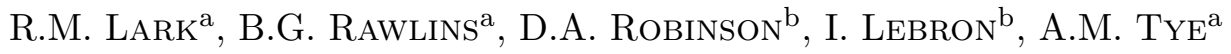 \\ ${ }^{\mathrm{a}}$ British Geological Survey, Keyworth, Nottingham, NG12 5GG, ${ }^{\mathrm{b}}$ Centre for Ecology and Hy- \\ drology, Environment Centre Wales, Deiniol Road, Bangor, Gwynedd LL57 2UW
}

Short title: Bulk density sampling

Key words: Bulk density; sampling; protocols; pedotransfer functions; soil organic carbon

Correspondence: R.M. Lark. E-mail: mlark@nerc.ac.uk 


\section{Summary}

Soil bulk density (BD) is measured in soil monitoring. Because it is spatially variable, an appropriate sampling protocol is required. It is shown how information on short-range variability can be used to quantify uncertainty of estimates of mean BD and soil organic carbon on a volumetric basis $\left(\mathrm{SOC}_{\mathrm{v}}\right)$ at a sampling site with different sampling intensities. We report results from two contrasting study areas, with mineral soil and with peat. More sites should be investigated to develop robust protocols for national-scale monitoring, but these results illustrate the methodology. A $20 \times 20-\mathrm{m}$ monitoring site was considered and sampling protocols were evaluated under geostatistical models of our two study areas. On sites with local soil variability comparable to our mineral soil, sampling at 16 points ( $4 \times 4$ square grid of interval $5 \mathrm{~m})$ would achieve a root mean square error (RMSE) of the sample mean value of both $\mathrm{BD}$ and $\mathrm{SOC}_{\mathrm{v}}$ less than $5 \%$ of the mean (top-soil and sub-soil). Pedotransfer functions (PTFs) gave predictions of mean soil BD at a sample site, comparable to our study area on mineral soil, with similar precision to a single direct measurement of $\mathrm{BD}$.

On peat soils comparable to our second study area, the mean BD for the monitoring site at depth 0-50 cm would be estimated with RMSE less than $5 \%$ of the mean with a sample of 16 cores, but at greater depths this criterion cannot be achieved with 25 cores or fewer. 


\section{Introduction}

Bulk density (BD) is a fundamental property of the soil. For purposes of this paper BD is the mass per unit volume of oven-dried soil material, after exclusion of stones of diameter $>$ $2 \mathrm{~mm}$. Bulk density is an indicator of soil quality because good management, that enhances soil structure, porosity and organic content, will tend to reduce $\mathrm{BD}$; conversely $\mathrm{BD}$ is increased if soil is compacted and loses its structure (Schipper \& Sparling, 2000; Black et al., 2008). Furthermore, the BD of the soil must be known if analytical data that have been determined on a mass basis, as is standard practice for variables such as soil organic carbon (we refer to the soil organic content per unit mass as $\mathrm{SOC}_{\mathrm{m}}$ ), are to be converted to a volume basis (we refer to the soil organic content per unit volume as $\mathrm{SOC}_{\mathrm{v}}$ ) and so to estimates of total stock per unit area. The $\mathrm{BD}$ of soil is commonly used as a predictor variable in pedotransfer functions to predict hard-to-measure properties of the soil such as parameters of the water retention curve or of the unsaturated hydraulic conductivity function (Schapp et al., 2001). For all these reasons it is generally accepted that BD should be measured as part of soil inventory or monitoring (Black et al., 2008). This paper addresses the question of how a single monitoring site should be sampled to arrive at a value of $\mathrm{BD}$.

Bulk density is more laborious to measure than many soil properties because a soil specimen of known volume must be extracted by a procedure that causes minimal disturbance. It is therefore necessary to sample BD efficiently. If we are to chose an appropriate sampling strategy to estimate the $\mathrm{BD}$ of soil at a monitoring site then we must consider how variable $\mathrm{BD}$ is within a site, and we must know how much error is tolerable in the final estimate. Error in the estimated $\mathrm{BD}$ at a monitoring site will propagate, inflating the error of the determinations of soil composition on a volume basis. The tolerable error in BD therefore depends on the tolerable error in these volumetric data.

Soil scientists need to know what constitutes an appropriate strategy for determining soil $\mathrm{BD}$ at a monitoring site. In particular, how many determinations should be made at a site, considering both the acceptable error in $\mathrm{BD}$ and in volumetric compositional data which are 
computed with the $\mathrm{BD}$ value? One strategy (Black et al., 2008) is to make a single determination of $\mathrm{BD}$ at one point in a monitoring site, and to use this value as representative when determining volumetric concentrations from gravimetric data obtained by analysing aggregate material from different locations within the monitoring site. This approach should be evaluated. Since BD is laborious to determine in the field, and is not available for some historical soil inventories in the UK (SNIFFER, 2007), one might also ask whether a prediction made with a pedotransfer function $(\mathrm{PTF})$ is an acceptable substitute for a direct measurement.

In this paper we demonstrate how geostatistical models of the spatial variability of soil properties at short-range (within the sampling site) can be used to compute the variances of sample means for both soil $\mathrm{BD}$ and soil organic carbon content on a volume basis $\left(\mathrm{SOC}_{\mathrm{v}}\right)$, the latter depending in part on the sample error of $\mathrm{BD}$, under different sampling strategies. This allows one to compute how the sample variances depend on the number of cores which are collected and on which of these cores $\mathrm{BD}$ or $\mathrm{SOC}_{\mathrm{m}}$ or both are determined. Note that, while this research is focussed on the determination of $\mathrm{BD}$ and volumetric composition of the soil, the same general approach could be used to determine sampling requirements for other properties of the soil.

In this paper we report research in which we examined the spatial variability of soil BD over short distances at two study areas, one with predominantly organic soils and the other with mineral soils. These sites were selected as, respectively, typical examples of upland organic soils from the west of Great Britain, and inorganic soils in arable use in the East Midlands of England. However, the results that we present should not be treated as a basis for generalization about the sampling requirements on all mineral or organic soils. Further work, using the methodology developed and reported here, and geostatistical models of short-range soil variability obtained using similar methods to this study across a wider range of study areas, is needed to develop robust sampling protocols. Given this information, and subject to the noted caveats, it was then possible to show the implications of the observed short-range variation of $\mathrm{BD}$ for sampling in the study areas. In particular: 
(i) How does the error in the BD estimate for a sample site respond to increased sample effort? This question was addressed for both mineral and organic soils

(ii) How does the error in $\mathrm{SOC}_{\mathrm{v}}$, determined directly by measuring $\mathrm{SOC}_{\mathrm{m}}$ and $\mathrm{BD}$ on each of a set of cores, respond to increased sample effort? This question was addressed for mineral soils.

(iii) How much error must be accepted for determinations of $\mathrm{SOC}_{\mathrm{v}}$ at a site if a determination of $\mathrm{SOC}_{\mathrm{m}}$ from an aggregate sample is combined with a single measurement of $\mathrm{BD}$ at an independent location at the site? This question was addressed for mineral soils.

(iv) How much error must be accepted for determinations of $\mathrm{BD}$ and of $\mathrm{SOC}_{\mathrm{v}}$ at a site if $\mathrm{BD}$ is not determined directly but rather is predicted by a PTF? This question was addressed for mineral soils.

\section{Materials and methods}

In this project we consider a monitoring site to be a square area of length $20 \mathrm{~m}$. This coincides with practice in the National Soil Inventory (NSI) of England and Wales, the Geochemical Baseline of the United Kingdom (SNIFFER, 2007) and recommendations for a UK-wide soil monitoring scheme made by Black et al. (2008). We describe first how two study areas were sampled to provide information on variability of soil properties at scales up to $20 \mathrm{~m}$. We then describe the estimation of parameters for a PTF to predict BD of mineral soils from archival data. We then describe spatial analyses of the resulting data to address the questions enumerated in the introduction.

\section{Field sampling}

Organic soil site This site was at the Nant-y-Brwyn catchment in Snowdonia, Wales (Latitude= $52.99510,^{\circ} \mathrm{N}$, Longitude $=3.80285^{\circ} \mathrm{W}$, mean altitude $\left.440 \mathrm{~m}\right)$. These organic soils are Histosols according to the WRB classification (IUSS Working Group WRB, 2006) and were mapped within the Crowdy 1 Soil Association by the Soil Survey of England and Wales (1984a). This 
association is dominated by the Crowdy series (amorphous raw peat), with some stagnohumic gleys and stagnopodzols (National Soil Resources Institute, 2013). Land cover at this site is referred to as 'Bog' in the classification used for the Land Cover Map of Great Britain, (Fuller et al., 2002).

Available resources allowed for the collection and analysis of 75 soil cores. The objective of sampling was to estimate the variance parameters of a linear mixed model (LMM) of the data (Stein, 2000; Lark et al., 2006a). We therefore decided to use purposive sampling on transects, with clusters of sample points within which the variability of soil properties at lag distances up to $20 \mathrm{~m}$ could be observed. Ten such clusters were arranged on four transects. The transects were selected along routes where the soil could be sampled to at least $1 \mathrm{~m}$ depth, and where it was not affected by grips, drainage channels traditionally dug in the peat. The transects and sample clusters were laid out in the field by tape measure. The locations of the first and last point in each cluster were obtained with a differential GPS and the coordinates of the points within the clusters were then inferred. Figure 1 shows the distribution of the sample points.

At each sample location the soil was sampled with a Russian auger with a flight of length $50 \mathrm{~cm}$ and an estimated sample volume of $622 \mathrm{~cm}^{2}$. Samples were collected up to depth $2 \mathrm{~m}$. The samples were cut into $10-\mathrm{cm}$ sections (volume $124.4 \mathrm{~cm}^{2}$ ), and each section was placed in a pre-weighed bag. On return to the laboratory the bags were weighed then opened and placed in an oven to dry at $105^{\circ} \mathrm{C}$ for 72 hours. After drying the core sections were then reweighed. From these measurements the dry BD was computed for each 10-cm section. Organic carbon content was determined on material from each section by loss on ignition according to Countryside Survey protocols (Emmett et al., 2008) but with total time in ignition extended to 20 hours to ensure complete combustion of any wood. Data on organic carbon content were not used in the work reported here, except to report the organic status of the soils.

Mineral soil site This site was a field at the University of Nottingham's farm at Bunny in Nottinghamshire, England (Latitude $=52.8547^{\circ} \mathrm{N}$, Longitude $=1.1274^{\circ} \mathrm{W}$, mean altitude $39 \mathrm{~m}$ ). The soil of the field is a Luvisol in the WRB classification (IUSS Working Group WRB, 2006) 
and is mapped in the Dunnington Heath Association by the Soil Survey of England and Wales (1984b). This association is dominated by the Dunnington Heath Series, argillic brown earths of loamy or clayey texture with clay-enriched sub-soil (National Soil Resources Institute, 2013). The soil of this field is cultivated to depth $10 \mathrm{~cm}$ and is occasionally sub-soiled to depth $25 \mathrm{~cm}$. In recent years prior to sampling the field from which soil samples were collected had been under a winter wheat-oil seed rape rotation.

The field was sampled at 90 sample points on three transects. As at Nant-y-Brwyn the samples were distributed in clusters along the transects, here there were three clusters. The distribution of sample points is shown in Figure 2. The sample sites were surveyed prior to sampling with a measuring tape and marked with canes, then locations were obtained with a differential GPS. A soil core, diameter $55 \mathrm{~mm}$, was collected to depth $1 \mathrm{~m}$ with a sonic drill rig. Sonic drilling uses intense vibrations directed down the drill string so that intact soil can be extracted above a cutting shoe. This enables extremely rapid soil penetration with relatively light drilling equipment (Environmental Sampling Limited, Godstone, Surrey). After extraction the cores were transported upright in their liners and kept in a cold store.

The 90 cores from the principal sampling points were then cut into seven 5 -cm sections for depth intervals (i) $2.5-7.5 \mathrm{~cm}$, (ii) $7.5-12.5 \mathrm{~cm}$, (iii) $12.5-17.5 \mathrm{~cm}$, (iv) $17.5-22.5 \mathrm{~cm}$, (v) $32.5-$ $37.5 \mathrm{~cm}$, (vi) $47.5-52.5 \mathrm{~cm}$ and (vii) $72.5-77.5 \mathrm{~cm}$. For purposes of this paper we worked with the soil in the $2.5-7.5 \mathrm{~cm}$ and $32.5-37.5 \mathrm{~cm}$ depth intervals, and for convenience we refer to these as the top-soil and sub-soil hereafter.

The material was oven dried, sieved to pass $2 \mathrm{~mm}$ and the resulting dry fine-fraction material was weighed. In addition, the coarse material retained by the sieves was weighed and its volume was measured by displacement. The $\mathrm{BD}$ of the fine-fraction was then computed as the oven-dry mass of the fine-fraction divided by the volume of the fine-earth fraction in the field. This latter volume was calculated by subtracting the volume of the material that did not pass the sieve from the volume of the section. The resulting BD is that of the fine fraction (Hall et al., 1977). A 10-g subsample of fine-fraction material was taken from each of the top-soil 
(2.5-7.5-cm depth) and sub-soil (32.5-37.5-cm depth) sections and the organic carbon content was determined by loss on ignition. Although there is evidence that LOI can over-estimate the quantity of organic matter in a soil sample because of loss of structural water from clay minerals the magnitude of this effect is generally considered to be small (Soon \& Abbound, 1991).

\section{Development of a pedotransfer function}

In research allied to that reported in this paper, work was done to develop a PTF to predict BD of mineral soils in England and Wales. Here we explain the development of PTFs for top-soil and sub-soil BD that could be compared in terms of precision with direct measurements of $\mathrm{BD}$ or $\mathrm{SOC}_{\mathrm{v}}$ at a monitoring site. We considered a range of different functional forms for the PTFs, based on those reported in the literature, and estimated their parameters, and compared their goodness of fit using an available data set on soils of England and Wales.

The data used to fit PTFs were the SOILPITs data set, part of the LandIS information system held by the National Soil Resources Institute. These measurements include observations from more than one horizon of a single soil pit with determinations of $\mathrm{BD}$ (fine-fraction), $\mathrm{SOC}_{\mathrm{m}}$ and particle size distribution. We sorted the observations into shallow (horizon mid-depth less than $25 \mathrm{~cm}$ depth), of which there were 562 observations, and deep (mid-depth greater than $25 \mathrm{~cm}$ ), of which there were 440. Prediction data sets, 365 shallow observations and 284 deep, were selected by simple random sampling, to be used to fit PTFs for the two depth intervals.

All the observations were overlaid on the British Geological Survey's Parent Material Map of the British Isles (British Geological Survey, 2006) at 1:50 000 scale, and the Centre for Ecology and Hydrology's Land Cover Map 2000 (Fuller et al., 2007) for 1-km pixels of Great Britain. Parent Material Classes at the parent material origin level of the classification, and Land Use classes at the level of dominant broad habitats in each $1 \mathrm{~km}$ square were extracted for each soil profile observation.

For purposes of this paper we consider only PTFs with soil organic carbon as a predictor of BD. This is because we did not have sufficient resources to undertake particle size analysis of soil from all 90 sample sites. We fitted PTFs as LMM using the NLME library (Pinheiro et al., 
2012) for the $\mathrm{R}$ platform (R Development Core Team, 2012). This was necessary because the SOILPITS data set was not assembled by probability sampling. We compared different models using the log likelihood ratio statistic (Verbeke \& Molenberghs, 2000). We considered models in which the only predictor was some function of $\mathrm{SOC}_{\mathrm{m}}$, and models in which land use or parent material (PM) as described above were categorical predictors, either additive or interactive with $\mathrm{SOC}_{\mathrm{m}}$.

The best-fitting model to predict $\mathrm{BD}$ of the shallow soil samples corresponded to one proposed by Alexander (1980)

$$
D b_{\mathrm{f}}=\beta_{0}+\beta_{1} C^{1 / 2} \text {, }
$$

where $D b_{\mathrm{f}}$ denotes bulk density of the fine fraction, $C$ is $\mathrm{SOC}_{\mathrm{m}}$ and the coefficients $\beta_{0}$ and $\beta_{1}$ were estimated from the SOILPITS data as described above. Other studies have shown that this is an effective PTF; de Vos et al. (2005) found it to be the best-fitting model in a study of a large data set from Belgium. There was no benefit from including PM or land use as predictors. However, for the deep samples the best-fitting PTF included land use as a predictor, interacting with $C^{1 / 2}$, that is there are different intercepts and slopes for the regression of $D b_{\mathrm{f}}$ on $C^{1 / 2}$.

The BD of the fine fraction for each section collected at Bunny farm was predicted from $\mathrm{SOC}_{\mathrm{m}}$ using the PTF for the shallow soils to predict for the top-soil sections, and the PTF for the deep soils to predict for the sub-soil sections. The value of $\mathrm{SOC}_{\mathrm{v}}$ of each section was also predicted from the PTF prediction of $\mathrm{BD}$ and the measured $\mathrm{SOC}_{\mathrm{m}}$. This allowed us to compute, for each section, an error in the PTF-based prediction of $\mathrm{BD}$ and $\mathrm{SOC}_{\mathrm{v}}: \varepsilon_{\mathrm{PTF}, \mathrm{BD}}$ and $\varepsilon_{\mathrm{PTF}, \mathrm{SOCv}}$ respectively.

\section{Data analysis}

Summary statistics were computed for the data and, where necessary, these were transformed. Summary statistics are also presented for the $\mathrm{SOC}_{\mathrm{m}}$ data after transformation to square-roots. The data on BD were then analysed with a LMM

$$
\mathbf{z}=\mathbf{X} \boldsymbol{\beta}+\boldsymbol{\eta}+\boldsymbol{\epsilon}
$$


where $\mathbf{z}$ is an $n \times 1$ vector of $n$ observations, $\mathbf{X}$ is a design matrix which contains fixed effects, predictor variables for the dependent variable, $\boldsymbol{\beta}$ is a vector of fixed effects coefficients, $\boldsymbol{\eta}$ is a random variable which has a second-order stationary spatial covariance function and $\epsilon$ is an independently and identically distributed random variable. More detail on the LMM is provided by Lark et al., (2006a). In this study the only fixed effect that we considered was a constant mean. We used the LIKFIT procedure from the GEOR library (Ribeiro \& Diggle, 2001) for the R platform (R Development Core Team, 2012) to fit the model by residual maximum likelihood. Spherical and exponential covariance functions were considered. The key parameters to estimate were the variances of the correlated and uncorrelated fixed effects, $\boldsymbol{\eta}$ and $\boldsymbol{\epsilon}$, which are $c_{1}$ and $c_{0}$ respectively, and the distance parameter of the covariance function.

We then used the GSTAT library (Pebesma, 2004) for the R platform to estimate auto- and cross-variograms for $\mathrm{BD}$ (transformed where necessary) and square root of the concentration of $\mathrm{SOC}_{\mathrm{m}}$ (mineral soil data) and fitted a linear model of coregionalization (LMCR, Journel \& Huijbregts, 1978) using the procedure of Lark \& Papritz (2003). The reader is referred to the cited literature for more detail in the LMCR. In short, the model comprises one or more authorised variogram functions (such as the exponential or spherical) with distance parameters used to model jointly the variograms and cross-variogram(s) of two or more variables with variances and covariances to ensure a positive definite covariance matrix (also known as the coregionalization matrix) for each included variogram. We estimated variograms and fitted an LMCR using the square root of $\mathrm{SOC}_{\mathrm{m}}$ because, as is shown in the literature (de Vos et al., 2005), this makes the assumption of a linear coregionalization of these variables most plausible.

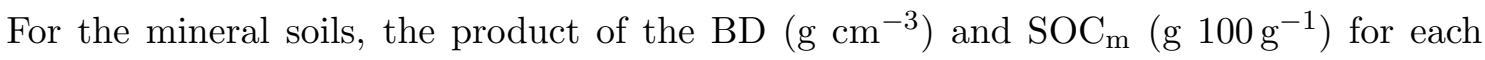
section was multiplied by ten to give a value of $\mathrm{SOC}_{\mathrm{v}}\left(\mathrm{mg} \mathrm{C} \mathrm{cm}^{-3}\right)$. Summary statistics of this variable were calculated, and a LMM was fitted, as for the BD data.

Summary statistics were computed of the errors of predictions with PTFs of BD for each section, $\varepsilon_{\mathrm{PTF}, \mathrm{BD}}$, (top-soil and sub-soil) from Bunny farm and the errors of predictions of $\mathrm{SOC}_{\mathrm{v}}$ based on these PTF-predictions, $\varepsilon_{\mathrm{PTF}, \mathrm{SOCv}}$. 


\section{Inference}

Precision of estimates of $B D$ and $S O C_{\mathrm{v}}$ by direct sampling. Our objective here is to quantify the uncertainty of values of $\mathrm{BD}$ and $\mathrm{SOC}_{\mathrm{v}}$ formed by sampling a $20 \times 20-\mathrm{m}$ monitoring site with different levels of effort. We consider systematic sampling, with cores collected on a regular grid. The location of the centre of the grid is fixed at the selected coordinates of the sample site, so there is no scope to think of the location of the grid as randomized within the sample site. The value of $\mathrm{BD}$ or $\mathrm{SOC}_{\mathrm{v}}$ recorded for the sample site is the arithmetic mean of the values for the individual cores, whether these are determined individually or aggregated. We consider sampling with a single core fixed at the centre of the monitoring site $(n=1)$, two cores $10 \mathrm{~m}$ apart and each $5 \mathrm{~m}$ from the centre of the monitoring site $(n=2)$ and $n=4,9,16$ or 25 cores on regular square grids with nodes at the centres of regular square tiles. The sample arrays are illustrated in Figure 3.

The uncertainty of the estimate of a mean value of a property across a monitoring site is quantified by a root mean square error. This is the square root of $S_{\mathrm{p}}^{2}$, the expected squared prediction error of the sample mean as a prediction of the spatial mean of the target variable across the $20 \times 20-\mathrm{m}$ monitoring site. This quantity is evaluated over the statistical model of the random effects in Equation (2), the fitting of which is described earlier. For untransformed variables we used the expression from Webster \& Oliver (1990)

$$
S_{\mathrm{p}}^{2}=\frac{2}{n} \sum_{i=1}^{n} \bar{\gamma}\left(\mathbf{x}_{i}, \mathcal{B}\right)-\frac{1}{n^{2}} \sum_{i=1}^{n} \sum_{j=1}^{n} \gamma\left(\mathbf{x}_{i}, \mathbf{x}_{j}\right)-\bar{\gamma}(\mathcal{B}, \mathcal{B})
$$

where $\mathbf{x}_{i}$ is a vector that denotes the location of the $i$ th out of $n$ cores, $\mathcal{B}$ denotes the $20 \times 20-\mathrm{m}$ monitoring site,

$$
\bar{\gamma}\left(\mathbf{x}_{i}, \mathcal{B}\right)=\int_{\mathbf{x}_{k} \in \mathcal{B}} \gamma\left(\mathbf{x}_{i}, \mathbf{x}_{k}\right) \mathrm{d} \mathbf{x}_{k}
$$

and

$$
\bar{\gamma}(\mathcal{B}, \mathcal{B})=\int_{\mathbf{x}_{k} \in \mathcal{B}} \int_{\mathbf{x}_{l} \in \mathcal{B}} \gamma\left(\mathbf{x}_{k}, \mathbf{x}_{l}\right) \mathrm{d} \mathbf{x}_{l} \mathrm{~d} \mathbf{x}_{k}
$$

where the integrals are over the two-dimensional space of $\mathcal{B}$.

If the variable was transformed, then $S_{\mathrm{p}}^{2}$ was computed numerically. The estimated vari- 
ance parameters of the transformed variable were used to simulate a set of values of that variable at points corresponding to (i) the sample sites and (ii) a set of 3000 additional points selected from across the region by simple random sampling. Simulation was done by the LU method (Goovaerts, 1997). The simulated values were back-transformed to the original units of measurement and then those corresponding to the sample sites were used to obtain a sample mean for the spatial mean across the sample region, and those at the 3000 additional points were used to form a very precise estimate of that spatial mean. The difference between the two means was recorded. This was repeated 5000 times, and the mean square difference between the two means over all iterations was treated as an estimate of $S_{\mathrm{p}}^{2}$.

We used these methods to compute mean square errors for site mean values of $\mathrm{BD}, \mathrm{SOC}_{\mathrm{v}}$ and $\mathrm{SOC}_{\mathrm{m}}\left(S_{\mathrm{p}, \mathrm{BD}}^{2}, S_{\mathrm{p}, \mathrm{SOCv}}^{2}\right.$ and $S_{\mathrm{p}, \mathrm{SOCm}}^{2}$ respectively) for different sampling grids.

Precision of estimates of $B D$ and $S O C_{\mathrm{v}}$ by PTFs. One way to estimate the mean $\mathrm{BD}$ at a monitoring site is to predict it with a PTF from the mean $\mathrm{SOC}_{\mathrm{m}}$. The predicted $\mathrm{BD}$ could then be used to estimate the mean $\mathrm{SOC}_{\mathrm{v}}$. There are three sources of error in this prediction. The first is bias in the PTF, the second is imprecision in the PTF and the third is estimation error in the value of $\mathrm{SOC}_{\mathrm{m}}$ used as the predictor variable. If we treat these as three independent error sources then we could write a mean squared error for predicted BD as

$$
S_{\mathrm{PTF}, \mathrm{BD}}^{2}=\left\{\bar{\varepsilon}_{\mathrm{PTF}, \mathrm{BD}}\right\}^{2}+\widehat{\operatorname{Var}}\left\{\varepsilon_{\mathrm{PTF}, \mathrm{BD}}\right\}+S_{\mathrm{p}, \mathrm{SOCm}}^{2}\left\{\frac{\partial}{\partial C} f(C)\right\}^{2}
$$

where the overbar in the first term denotes the mean, $\widehat{\operatorname{Var}}$ denotes the sample variance of the term in braces and $f(C)$ represents the $\mathrm{PTF}$ for $\mathrm{BD}$ with $C$ the predictor variable, $\mathrm{SOC}_{\mathrm{m}}$. The first term is the effect of bias in the PTF and the second term is the effect of imprecision. The third term is the effect of sampling error in the value of $\mathrm{SOC}_{\mathrm{m}}$, and is calculated from a first-order Taylor series approximation to the PTF (Heuvelink, 1998), this was evaluated at the mean value of $\mathrm{SOC}_{\mathrm{m}}$. Clearly the value of $S_{\mathrm{PTF}, \mathrm{BD}}^{2}$ depends on the sampling configuration used to estimate the mean value of $\mathrm{SOC}_{\mathrm{m}}$. A similar calculation can be made for the mean square 
error of determinations of $\mathrm{SOC}_{\mathrm{v}}$ based on the PTF-prediction of $\mathrm{BD}$. This is

$$
S_{\mathrm{PTF}, \mathrm{SOCv}}^{2}=\left\{\bar{\varepsilon}_{\mathrm{PTF}, \mathrm{SOCv}}\right\}^{2}+\widehat{\operatorname{Var}}\left\{\varepsilon_{\mathrm{PTF}, \mathrm{SOCv}}\right\}+S_{\mathrm{p}, \mathrm{SOCm}}^{2}\left\{\frac{\partial}{\partial C} 10 C f(C)\right\}^{2} .
$$

The value 10 appears in the last term because the $\mathrm{SOC}_{\mathrm{v}}$ values are scaled to $\mathrm{mg} \mathrm{C} \mathrm{cm}^{-3}$.

Precision of estimates of $S O C_{\mathrm{v}}$ by indirect sampling. Finally, we considered a strategy to estimate $\mathrm{SOC}_{\mathrm{v}}$ for a monitoring site by making a single measurement of $\mathrm{BD}$, and determining $\mathrm{SOC}_{\mathrm{m}}$ from an aggregate sample of some number of cores collected on the sample grids shown in Figure 3. This strategy might be favoured for practical reasons. There are advantages in determining a property like $\mathrm{SOC}_{\mathrm{m}}$ on an aggregate sample (Lark, 2011). However, the collection of a soil sample to determine $\mathrm{BD}$ is more laborious than the collection of cores for gravimetric determination of soil composition, since in the former case it is important to know the volume of the original sample, and to determine the dry mass of the fine fraction of the sample in its entirety. This approach is proposed by Black et al. (2008) in a national soil monitoring strategy for the UK.

We used a numerical method to estimate the mean square error of such an indirect determination of soil $\mathrm{SOC}_{\mathrm{v}}, S_{\mathrm{I}, \mathrm{SOCv}}^{2}$. This made use of the LMCR for soil BD (possibly transformed) and the square-root of $\mathrm{SOC}_{\mathrm{m}}$. The LMCR can be used to specify a covariance matrix for BD and square-root $\mathrm{SOC}_{\mathrm{m}}$ at a set of locations and this matrix, after LU decomposition, (Goovaerts, 1997) can be used to simulate joint values of $\mathrm{BD}$ and square-root $\mathrm{SOC}_{\mathrm{m}}$ at those locations. This method was used to generate a joint realization of $\mathrm{BD}$ and square-root $\mathrm{SOC}_{\mathrm{m}}$ at (i) one of the sets of grid sample points illustrated in Figure 3, (ii) a notional location for a BD measurement at a location close to the centre of the monitoring site and (iii) 3000 locations across the monitoring site selected by simple random sampling. From the simulated values of $\mathrm{SOC}_{\mathrm{m}}$ at the sample points we obtained an estimate of the spatial mean of $\mathrm{SOC}_{\mathrm{m}}$, and this was combined with the simulated value of $\mathrm{BD}$ at the single point near the centre of the site to provide an estimate of $\mathrm{SOC}_{\mathrm{v}}$. Both the simulated $\mathrm{SOC}_{\mathrm{m}}$ and $\mathrm{BD}$ values at the 3000 random locations were then used to provide a precise estimate of the spatial mean of $\mathrm{SOC}_{\mathrm{v}}$ for this particular realization. The error of the estimate based on the aggregate sample for $\mathrm{SOC}_{\mathrm{m}}$ and the single observation of $\mathrm{BD}$ 
could be computed. The mean square error was then calculated over 10000 realizations of the LMCR. As for the determinations of $S_{\mathrm{p}}^{2}$ described above, this provides us with a value for the expected square error of the estimate of the spatial mean over the statistical model that we have estimated for the joint distribution of the two variables.

\section{Results}

\section{Organic Soils from Nant-y-Brwyn}

The data on $\mathrm{BD}$ for the organic soils were very variable, and for this reason we aggregated the values into depth intervals of $50 \mathrm{~cm}$. Summary statistics for the BD data are given in Table 1, along with summary statistics for the data after transformation to natural logarithms. Note that the untransformed data for the $0-50 \mathrm{~cm}$ depth interval have a small coefficient of skewness, with an absolute value less than 0.5. In addition to the coefficient of skewness we computed the octile skew (Brys et al., 2003) which is a robust measure of skewness which is less susceptible to outliers than is the conventional skewness coefficient. Webster \& Oliver (2009) suggest as a rule of thumb that transformation to logarithms should be considered if the conventional skewness coefficient exceeds 1 , and a corresponding threshold for the octile skew is 0.2 (Lark et al., 2006b). On this criterion the data for the $0-50-\mathrm{cm}$ depth interval should be analysed in the original units, and the data at other depths should be transformed. Table 1 also presents variance parameters from the LMM fitted to the data on BD for these soils, transformed where necessary.

The data on organic matter content of these soil samples, determined by loss on ignition, showed that most of the sections had more than $50 \%$ organic carbon content by mass and so would be classified as peat (Hodgson, 1976). This was the case for $94 \%$ of samples at depth 0-50 cm, $80 \%$ at depth $50-100 \mathrm{~cm}, 94 \%$ at $100-150 \mathrm{~cm}$ and $96 \%$ at depth $150-200 \mathrm{~cm}$.

Figure 4 shows the root mean square errors for estimation of $\mathrm{BD}$ of organic soils on the different sample grids illustrated in Figure 3. The solid line represents $5 \%$ of the mean and the broken line $10 \%$. The graphs show the challenge of estimating $\mathrm{BD}$ in these circumstances is greatest at depth. For the top $50 \mathrm{~cm}$ a RMSE error less than $10 \%$ of the mean was achieved with four sample points, and $5 \%$ with 16 or more points, but at greater depth even 25 sample 
points do not suffice to reduce the mean squared error to $10 \%$ of the mean BD. At all depths the additional improvement from sampling 25 rather than 16 points is small.

\section{Mineral soils at Bunny Farm}

Summary statistics for $\mathrm{BD}, \mathrm{SOC}_{\mathrm{m}}$ and $\mathrm{SOC}_{\mathrm{v}}$ are shown in Table 2, along with statistics for some of these variables after transformation. Note that all three variables appear more or less symmetrically distributed with small coefficients of skewness and octile skew, apart from BD at the 32.5-37.5 depth interval. These values are negatively skewed. We found a Box-Cox transformation for this variable:

$$
\begin{aligned}
y & =\frac{z^{\zeta}-1}{\zeta}, \quad \zeta \neq 0, \\
& =\log _{e}(z), \quad \zeta=0,
\end{aligned}
$$

We estimated the transformation parameter, $\lambda$ by maximum likelihood, using the BOxCOX procedure from the MAss package (Venables \& Ripley, 2002) for the R platform (R Development Core Team, 2012). The estimate of $\lambda$ was 4.26 . The summary statistics for this transformed variable are shown in Table 2. We also computed summary statistics for the square-root of $\mathrm{SOC}_{\mathrm{m}}$ which was used in an LMCR with $\mathrm{BD}$. Note that the $\mathrm{SOC}_{\mathrm{m}}$ data still seem reasonably symmetrically distributed on the square root scale. Variance parameters from the LMM fitted to the data on $\mathrm{BD}$ (after Box-Cox transformation for the sub-soil interval) and for the $\mathrm{SOC}_{\mathrm{m}}$ and $\mathrm{SOC}_{\mathrm{v}}$ data are also shown in the table. Table 3 presents parameters of the LMCR fitted to the data on $\mathrm{BD}$ (transformed for the sub-soil) and square root of $\mathrm{SOC}_{\mathrm{m}}$. Figure 5 shows the root mean square errors for estimation of mean $\mathrm{BD}$ of mineral soil at a monitoring site by direct sampling on grids of different intensity (solid discs) or by prediction with the PTF from the mean value of $\mathrm{SOC}_{\mathrm{m}}$ estimated from sample grids of different intensity (open circles). Note that, with direct sampling of $\mathrm{BD}$, the mean is reduced to less than $5 \%$ of the sample mean with a sample size of 4 (top-soil) or 9 (sub-soil). In contrast, the RMSE for PTF-based predictions of $\mathrm{BD}$ is always larger than $10 \%$ of the mean, and is not sensitive to reductions in the error variance of the mean of $\mathrm{SOC}_{\mathrm{m}}$ used as the predictor. 
Figure 6 shows the root mean square errors for estimation of the mean $\mathrm{SOC}_{\mathrm{v}}$ of mineral soil at a monitoring site by direct sampling on grids of different intensity (solid discs), by PTF prediction of $\mathrm{BD}$ from the mean $\mathrm{SOC}_{\mathrm{m}}$, then combined to estimate $\mathrm{SOC}_{\mathrm{v}}$ (open circles) or by combining an estimate of $\mathrm{SOC}_{\mathrm{m}}$ from an aggregated sample from the grid with a single measurement of $\mathrm{BD}$ (solid square). The direct measurement of $\mathrm{SOC}_{\mathrm{v}}$ allows the mean to be estimated with RMSE less than $5 \%$ of the sample mean with 9 (top-soil) or 16 (sub-soil) soil samples on a grid. Prediction via a PTF for BD does not give RMSE less than $10 \%$ of the mean for any of the sample sizes considered here. Note also that estimating mean $\mathrm{SOC}_{\mathrm{v}}$ from a single measurement of $\mathrm{BD}$ and independent observations of $\mathrm{SOC}_{\mathrm{m}}$ gives RMSE very similar to estimates based on the PTF, and only just less than $10 \%$ of the mean in the case of the top-soil.

\section{Discussion}

On the basis of these results we may make the following observations about the soils of the two study areas reported here. It is clear that the determination of BD in the peat soil requires considerably more sampling effort at depths below $50 \mathrm{~cm}$ than for the surface material. This reflects the very skewed distribution of $\mathrm{BD}$ for peat at the greater depths (as a result of which we used a transformation to logarithms). The RMSE of mean BD for a monitoring site can be reduced to less than $5 \%$ of the sample mean with a sample of 16 cores for depth $0-50 \mathrm{~cm}$, but at greater depths the improvement in RMSE with more than 16 cores is small, and the RMSE remains larger than $10 \%$ of the mean.

In the mineral soil rather less sample effort was required in the top-soil than the sub-soil for measurement of $\mathrm{BD}$, but nine cores ensured an RMSE less than $5 \%$ of the mean at both depths. It is clear that prediction of $\mathrm{BD}$ with a $\mathrm{PTF}$ based on $\mathrm{SOC}_{\mathrm{m}}$ gives poorer estimates than direct sampling. In no case is the RMSE less than $10 \%$ of the sample mean, although the RMSE from a PTF prediction is similar to that for a single determination of BD in the monitoring site. This suggests that, if BD is to be measured in the field, then it is appropriate to make more than one determination at any depth, otherwise a PTF prediction may be just as good. 
To determine $\mathrm{SOC}_{\mathrm{v}}$ at a sample site in the mineral soil study area by direct measurement requires slightly more sample effort than to determine $\mathrm{BD}$, since there are two sources of uncertainty (BD and $\mathrm{SOC}_{\mathrm{m}}$ ) to contend with. However, a sample of 16 cores ensures an RMSE less than $5 \%$ of the mean at both depths, and 9 cores would suffice for the top-soil. It is notable that indirect estimation of $\mathrm{SOC}_{\mathrm{v}}$ from a single $\mathrm{BD}$ determination and independent measurements of $\mathrm{SOC}_{\mathrm{m}}$ has an RMSE comparable to that from PTF prediction. Our analyses show that this approach, which is proposed for the UK national soil monitoring scheme (Black et al. 2008), is sub-optimal. The results in Figure 6 show that a substantial improvement in RMSE would be achieved by making just two $\mathrm{BD}$ determinations with $\mathrm{SOC}_{\mathrm{m}}$ determined on a representative aliquot of the same material. At the least, if a single sample is to be taken to determine $\mathrm{BD}$, then the $\mathrm{SOC}_{\mathrm{m}}$ of the same material should be determined.

Once again, these specific results are for two contrasting study areas, one on organic soil and one on mineral soil. To form robust conclusions for practice at national scale it would be necessary to conduct similar sampling and analysis on additional sites, at least to include mineral soils with a wider range of textural classes and $\mathrm{SOC}_{\mathrm{m}}$ concentrations. This paper sets out the methods by which such a study should be conducted. That said, it is encouraging that the sampling effort indicated for the mineral soil example appears feasible (it is less intensive than the protocol used for the National Soil Inventory of England and Wales, with 25 cores per sample site). However, there may be concerns that the variability of peat soils at depth might make it difficult to achieve good data from monitoring sites without prohibitively intensive sampling. The results reported for the study area over mineral soil also indicate that PTF predictions of BD may compare unfavourably with direct observations of BD.

\section{Conclusions}

This study allows us to draw specific conclusions about sampling requirements for determination of $\mathrm{BD}$ and $\mathrm{SOC}_{\mathrm{v}}$ only for monitoring sites on soils comparable to those at our two sites. In particular, sampling a $20 \times 20-\mathrm{m}$ monitoring site over mineral soil at 16 points $(4 \times 4$ square grid of interval $5 \mathrm{~m}$ ) gives a mean value of $\mathrm{BD}$ and $\mathrm{SOC}_{\mathrm{v}}$ in the top-soil and sub-soil with an RMSE 
of less than $5 \%$ of the mean. A smaller sample of four points $(2 \times 2$ square grid of interval $10 \mathrm{~m})$ gives an RMSE less than $10 \%$ of the mean. On peat soils the mean BD for the monitoring site at depth $0-50 \mathrm{~cm}$ can be estimated with RMSE less than $10 \%$ of the mean with a sample of four cores, and less than $5 \%$ of the mean with a sample of 16 cores, but at greater depths these criteria cannot be achieved, even with 25 cores. How far these conclusions can be generalized over other mineral or organic soils remains to be seen and would require comparable studies across a wider range of study areas.

Some results from these two study areas would be of particular interest if they are found to hold generally. In particular, under the geostatistical model for the study area with mineral soil the use of PTFs to obtain the BD at a sample site gave results with comparable precision to a single measurement of $\mathrm{BD}$, and the RMSE is larger than $10 \%$ of the mean. If this is generally the case then it would suggest that, while they may be useful for inferring BD from legacy soil data, PTFs are not appropriate as a substitute for direct observation of BD in newly-planned inventory and monitoring. Similarly the determination of $\mathrm{SOC}_{\mathrm{v}}$ using a single measurement of $\mathrm{BD}$ and independent cores to determine mean $\mathrm{SOC}_{\mathrm{m}}$ gaves results with precision similar to those obtained with PTF prediction. If four or more cores are to be collected then the benefits of determining $\mathrm{BD}$ as well as $\mathrm{SOC}_{\mathrm{m}}$ may be substantial. This suggests that the proposed approach (Black et al., 2008) of using a single measurement of BD at each sample site to rescale gravimetric measurements to volumetric ones may not be satisfactory.

\section{Acknowledgments}

This research was funded by the Department for Environment, Food and Rural Affairs (Defra) under project SP1305 'Studies to inform policy development with regard to soil degradation (Subproject B, Determination of soil BD)'. The soILPITs data were licenced from the National Soil Resources Institute for use in this project. We are grateful to Nottingham University Farm staff for access to the field and for cooperation when planning field work. The authors acknowledge the contribution made by Daniel Romberg to work at the Nant-y-Brwyn site, and 
433 by Michael Strutt to laboratory work in Keyworth. This paper is published with the permission 434 of the Director of the British Geological Survey (NERC). 


\section{References}

Alexander, E.B. 1980. Bulk densities of California soils in relation to other soil properties. Soil Science Society of America Journal, 44, 689-692.

Black, H., Bellamy, P., Creamer, R., Elston, D., Emmett, B., Frogbrook, Z., Hudson, G. et al. 2008. Design and operation of a UK soil monitoring network. Science Report SC060073. Environment Agency, Bristol, UK.

British Geological Survey, 2006. Digital Geological Map of Great Britain 1:50 000 scale (DiGMapGB50) data [CD-ROM] Version 3.14. British Geological Survey, Keyworth, Nottingham.

Brys, G., Hubert, M. \& Struyf, A. 2003. A comparison of some new measures of skewness. In: Developments in Robust Statistics (eds R. Dutter, P. Filzmoser, U. Gather and P.J. Rousseeuw), pp. 98-113. Physica-Verlag, Heidelberg.

De Vos, B., Van Meirvenne, M., Quataert, P., Deckers, J. \& Muys, M. 2005. Predictive quality of pedotransfer functions for estimating bulk density of forest soils. Soil Science Society of America Journal, 69, 500-510.

Emmett, B. A., Frogbrook, Z. L., Chamberlain, P. M., Griffiths, R., Pickup, R., Poskitt, J. et al. 2008. Soils Manual. Countryside Survey Technical Report no 03/0\%. Centre for Ecology and Hydrology, Lancaster, UK.

Fuller, R.M., Smith, G.M., Sanderson, J.M., Hill, R.A. \& Thomson, A.G. 2002. The UK Land Cover Map 2000: construction of a parcel-based vector map from satellite images. Cartographic Journal, 39, 15-25.

Goovaerts, P. 1997. Geostatistics for Natural Resources Evaluation Oxford University Press, New York, USA.

Hall, D.G.M., Reeve, M.J., Thomasson, A.J. \& Wright, V.F. 1977. Water Retention, Porosity and Density of Field Soils. Soil Survey Technical Monograph No. 9. Soil Survey of England and Wales, Harpenden, UK. 
Heuvelink, G.B.M. 1998. Error Propagation in Environmental Modelling with GIS. Taylor and Francis, London.

Hodgson, J.M. (ed.) 1976. Soil Survey Field Handbook. Soil Survey Technical Monograph No. 5. Soil Survey of England and Wales, Harpenden.

IUSS Working Group WRB, 2006. World Reference Base for Soil Resources 2006. 2nd edition. World Soil Resources Reports No. 103. FAO, Rome, Italy.

Journel, A.G. \& Huijbregts, C.J. 1978. Mining Geostatistics. Academic Press, London, UK.

Lark, R.M. \& Papritz, A. 2003. Fitting a linear model of coregionalization for soil properties using simulated annealing. Geoderma, 115, 245-260.

Lark, R.M., Cullis, B.R. \& Welham, S.J. 2006a. On spatial prediction of soil properties in the presence of a spatial trend:- the empirical best linear unbiased predictor (E-BLUP) with REML. European Journal of Soil Science, 57, 787-799.

Lark, R.M, Bellamy, P.H. \& Rawlins, B.G. 2006b. Spatio-temporal variability of some metal concentrations in the soil of eastern England, and implications for soil monitoring. Geoderma, 133, 363-379.

National Soil Resources Institute (NSRI) 2013. The Soils Guide. Available: www.landis.org.uk. Cranfield University, UK. Last accessed 23/05/2014

Pebesma, E.J., 2004. Multivariable geostatistics in S: the gstat package. Computers $\&$ Geosciences, 30, 683-691.

Pinheiro, J., Bates, D., DebRoy, S., Sarkar, D. \& R Development Core Team. 2012. nlme: Linear and Nonlinear Mixed Effects Models. R package version 3.1-105.

R Development Core Team. 2012. R: A language and environment for statistical computing. R Foundation for Statistical Computing, Vienna, Austria. http://www.R-project.org/. 
Rawlins, B. G., Scheib, A., Lark, R. M. \& Lister, T. R. 2009. Sampling and analytical plus subsampling variance components for five soil indicators observed at regional scale. European Journal of Soil Science, 60, 740-747.

Ribeiro, P.J., Diggle, P.J. 2001. geoR: a package for geostatistical analysis. R-NEWS, 1, 15-18.

Schaap, M.G., Leij, F.J. \& van Genuchten, M.Th. 2001. RosetTA: A computer program for estimating soil hydraulic parameters with hierarchical pedotransfer functions. Journal of Hydrology, 251, 163-176.

Schipper, L.A. \& Sparling, G.P. 2000. Performance of soil condition indicators across taxonomic groups and land uses. Soil Science Society of America Journal, 64, 300-311.

SNIFFER, 2007. National Soil Monitoring Network: Review and Assessment Study. SNIFFER, Edinburgh, UK. LQ09, June 2007.

Soil Survey of England and Wales. 1984a Soils and their Use in Wales Soil Survey Bulletin No. 11.

Soil Survey of England and Wales. 1984b Soils and their use in Midland and Western England Soil Survey Bulletin No. 12.

Soon, Y.K. \& Abboud, S. 1991. A comparison of some methods for soil organic carbon determination. Communications in Soil Science E Plant Analysis, 22, 943-954.

Stein, M.L. 1999. Interpolation of Spatial Data: some Theory for Kriging. Springer, New York, USA.

Venables, W. N. \& Ripley, B. D. 2002. Modern Applied Statistics with S. 4th Edition. Springer, New York, USA.

Verbeke, G., Molenberghs, G. 2000. Linear Mixed Models for Longitudinal Data. Springer, New York, USA. 
Webster, R. \& Oliver, M.A. 1990. Statistical Methods in Soil and Land Resource Survey. Oxford University Press, Oxford, UK.

Webster, R. \& Oliver, M.A. 2009. Geostatistics for Environmental Scientists. 2nd Edition John Wiley \& Sons, Chichester, UK. 


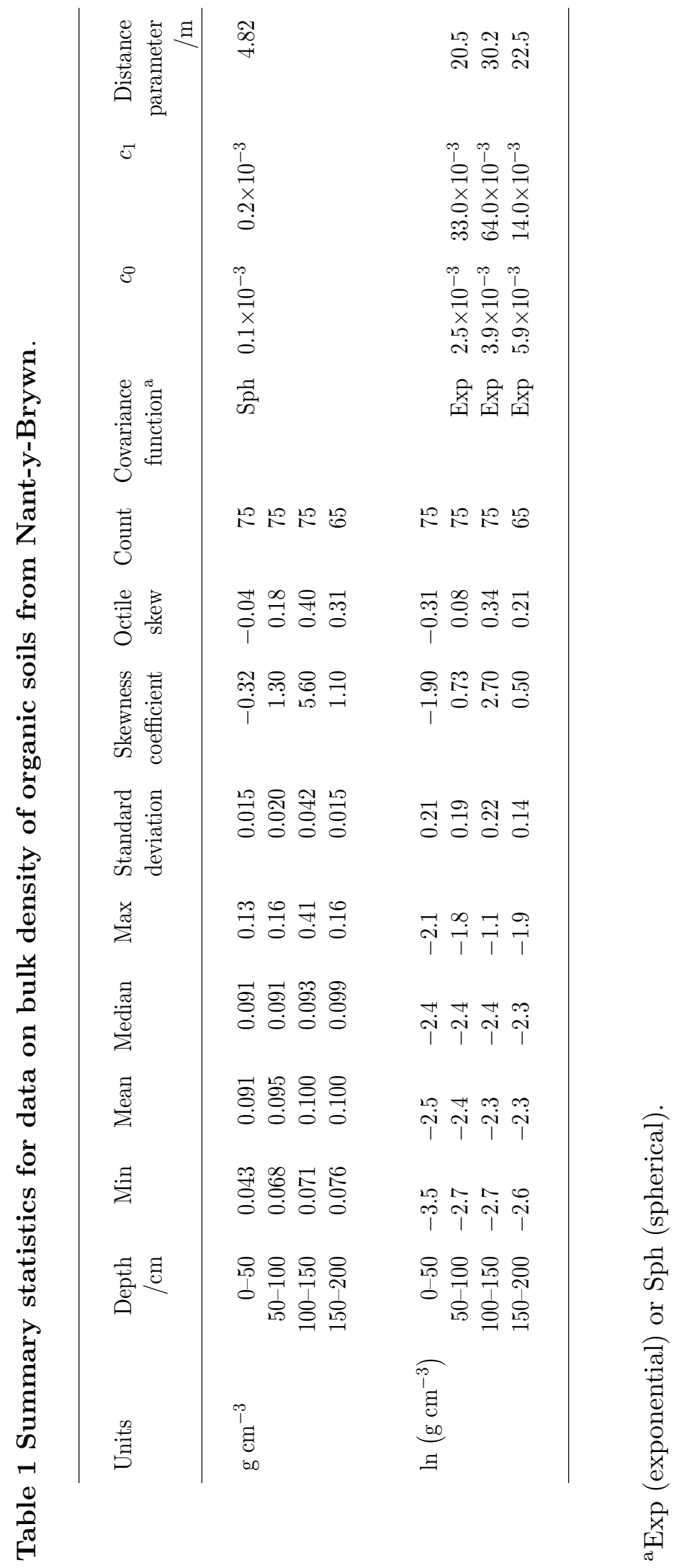




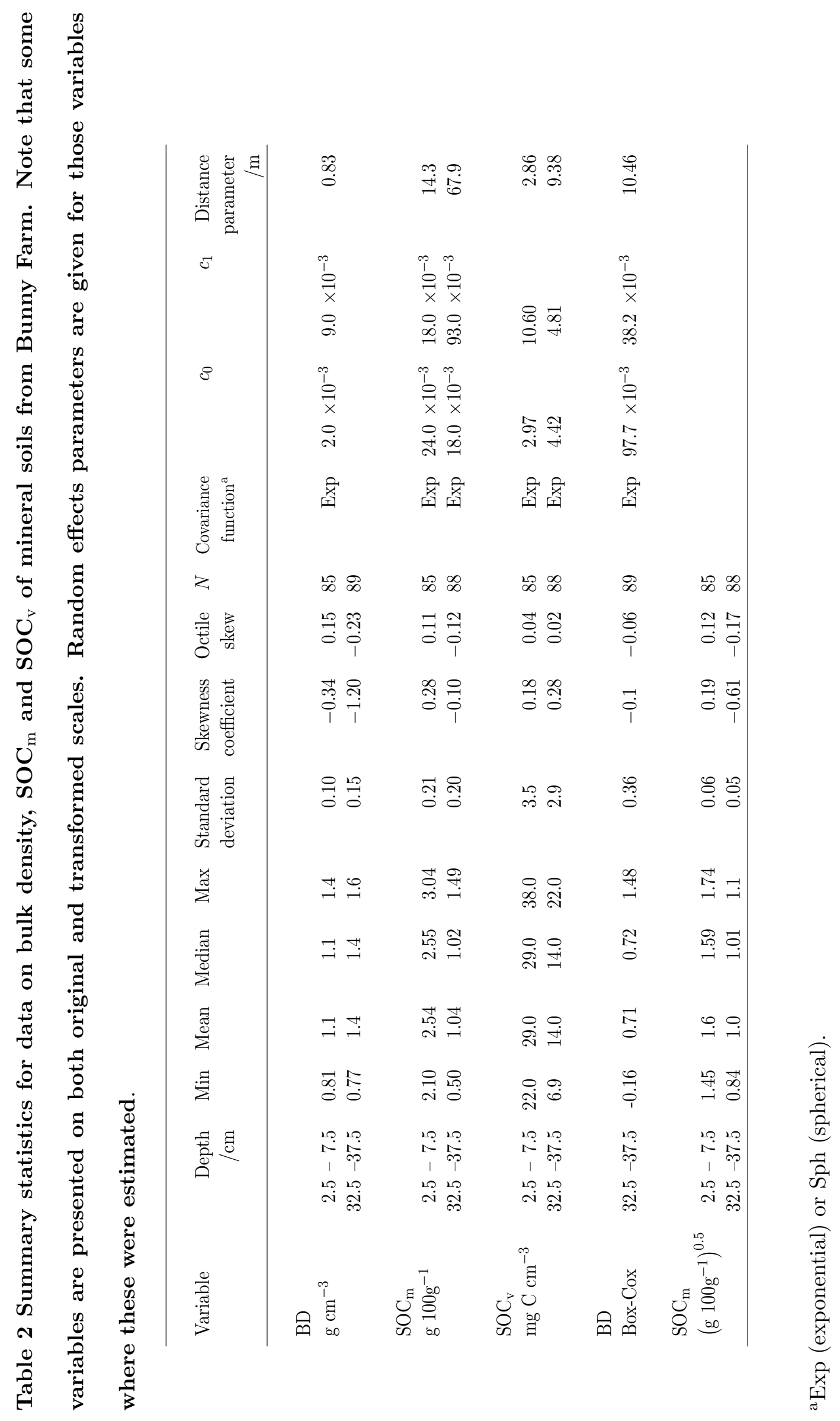


Table 3 Parameters of the linear models of coregionalization for (transformed) BD and square root of $\mathrm{SOC}_{\mathrm{m}}$.

\begin{tabular}{|c|c|c|c|c|}
\hline Variable & $\begin{array}{l}\text { Covariance } \\
\text { function }\end{array}$ & $c_{0}$ & $c_{1}$ & $\begin{array}{r}\text { Distance } \\
\text { parameter } \\
/ \mathrm{m} \\
\end{array}$ \\
\hline \multicolumn{5}{|l|}{ top-soil } \\
\hline $\begin{array}{l}\mathrm{BD} \\
\sqrt{\mathrm{SOC}_{\mathrm{m}}} \\
\mathrm{BD} \times \sqrt{\mathrm{SOC}_{\mathrm{m}}}\end{array}$ & Exponential & $\begin{array}{r}9.08 \times 10^{-3} \\
1.93 \times 10^{-3} \\
-1.15 \times 10^{-3}\end{array}$ & $\begin{array}{l}2.51 \times 10^{-3} \\
2.33 \times 10^{-3} \\
1.67 \times 10^{-3}\end{array}$ & 2.43 \\
\hline \multicolumn{5}{|l|}{ sub-soil } \\
\hline $\begin{array}{l}\text { Transformed }{ }^{\mathrm{a}} \mathrm{BD} \\
\sqrt{\mathrm{SOC}_{\mathrm{m}}} \\
\text { Transformed BD } \times \sqrt{\mathrm{SOC}_{\mathrm{m}}}\end{array}$ & Exponential & $\begin{array}{r}82.0 \times 10^{-3} \\
634.0 \times 10^{-6} \\
1.29 \times 10^{-3}\end{array}$ & $\begin{array}{r}69.0 \times 10^{-3} \\
10.4 \times 10^{-3} \\
-11.3 \times 10^{-3}\end{array}$ & 8.93 \\
\hline
\end{tabular}

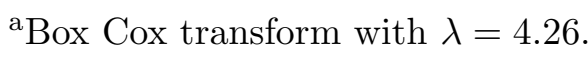


Table 4 Summary statistics for errors of predictions of BD by PTF, and errors of predictions of $\mathrm{SOC}_{\mathrm{v}}$ based on predicted BD, for mineral soils at Bunny Farm.

\begin{tabular}{|c|c|c|c|c|c|c|c|c|}
\hline Variable & $\begin{array}{r}\text { Depth } \\
/ \mathrm{cm} \\
\end{array}$ & Min & Mean & Median & Max & $\begin{array}{l}\text { Standard } \\
\text { deviation }\end{array}$ & $\begin{array}{c}\text { Skewness } \\
\text { coefficient }\end{array}$ & $\begin{array}{r}\text { Octile } \\
\text { skew }\end{array}$ \\
\hline \multicolumn{9}{|l|}{$\mathrm{BD}$} \\
\hline \multirow[t]{2}{*}{$\mathrm{g} \mathrm{cm}^{-3}$} & $2.5-7.5$ & -0.195 & 0.052 & 0.064 & 0.345 & 0.107 & 0.24 & -0.17 \\
\hline & $32.5-37.5$ & -0.223 & -0.029 & -0.050 & 0.547 & 0.143 & 1.20 & 0.13 \\
\hline \multirow[t]{2}{*}{$\begin{array}{l}\mathrm{SOC}_{\mathrm{v}} \\
\mathrm{mg} \mathrm{C} \mathrm{cm}\end{array}$} & $2.5-7.5$ & -9.44 & -1.290 & -1.510 & 4.82 & 2.75 & -0.32 & 0.11 \\
\hline & $32.5-37.5$ & -6.45 & 0.271 & 0.491 & 2.95 & 1.54 & -1.28 & -0.15 \\
\hline
\end{tabular}




\section{Figure Captions.}

1. Locations of sample points at the Nant-y-Brwyn site. Coordinates are in metres relative to the datum of the British National Grid.

2. Locations of sample points at the Bunny Farm site. Coordinates are in metres relative to the datum of the British National Grid.

3. Notional sample grids with $1,2,4,9,16$ or 25 sample points to characterize a $20 \mathrm{~m} \times 20-\mathrm{m}$ monitoring site.

4. Root mean square error of determinations of mean BD for different depths at a monitoring site (organic soil, statistics from the Nant-y-Brwyn data) by sampling on the grids in Figure 3. The broken and solid lines correspond to $10 \%$ and $5 \%$ of the sample mean of the Nant-Y-Brwyn data.

5. Root mean square error (RMSE) of determinations of mean BD for different depths at a monitoring site (mineral soil, statistics from the Bunny Farm data) by sampling on the grids in Figure 3. Solid discs are the RMSE of the mean of measurements of BD at each sample points. Open circles are $S_{\mathrm{PTF}, \mathrm{BD}}$, Equation (4) i.e. RMSE of the prediction of mean $\mathrm{BD}$ by using the mean value of $\mathrm{SOC}_{\mathrm{m}}$ from the sample points as the predictor in a PTF. The broken and solid lines correspond to $10 \%$ and $5 \%$ of the sample mean of the Bunny Farm data.

6. Root mean square error (RMSE) of determinations of mean $\mathrm{SOC}_{\mathrm{v}}$ for different depths at a monitoring site (mineral soil, statistics from the Bunny Farm data) by sampling on the grids in Figure 3. Solid discs are the RMSE of the mean of measurements of $\mathrm{SOC}_{\mathrm{v}}$ at each sample point. Open circles are $S_{\mathrm{PTF}, \mathrm{SOCv}}$, Equation (5) i.e. RMSE of the prediction of mean $\mathrm{SOC}_{\mathrm{v}}$ by using the mean value of $\mathrm{SOC}_{\mathrm{m}}$ from the sample points as the predictor in a PTF to obtain $\mathrm{BD}$, which is then used to compute $\mathrm{SOC}_{\mathrm{v}}$. Solid squares are $S_{\mathrm{I}, \mathrm{SOC}}$ i.e. RMSE of the prediction of mean $\mathrm{SOC}_{\mathrm{v}}$ from a single determination of $\mathrm{BD}$ near the centre 
of the monitoring site and the mean $\mathrm{SOC}_{\mathrm{m}}$ from cores at the sample points. The broken and solid lines correspond to $10 \%$ and $5 \%$ of the sample mean of the Bunny Farm data. 

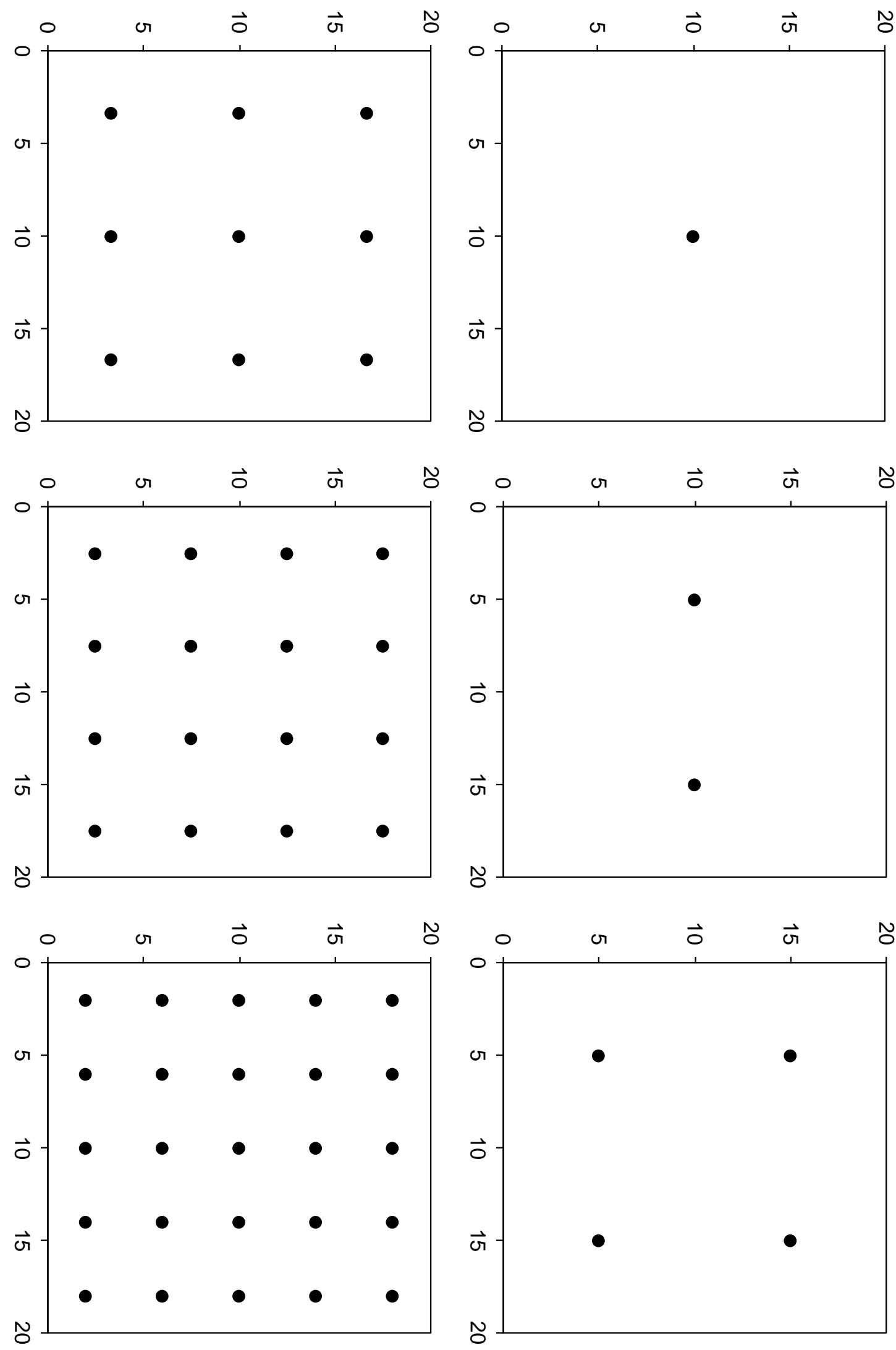

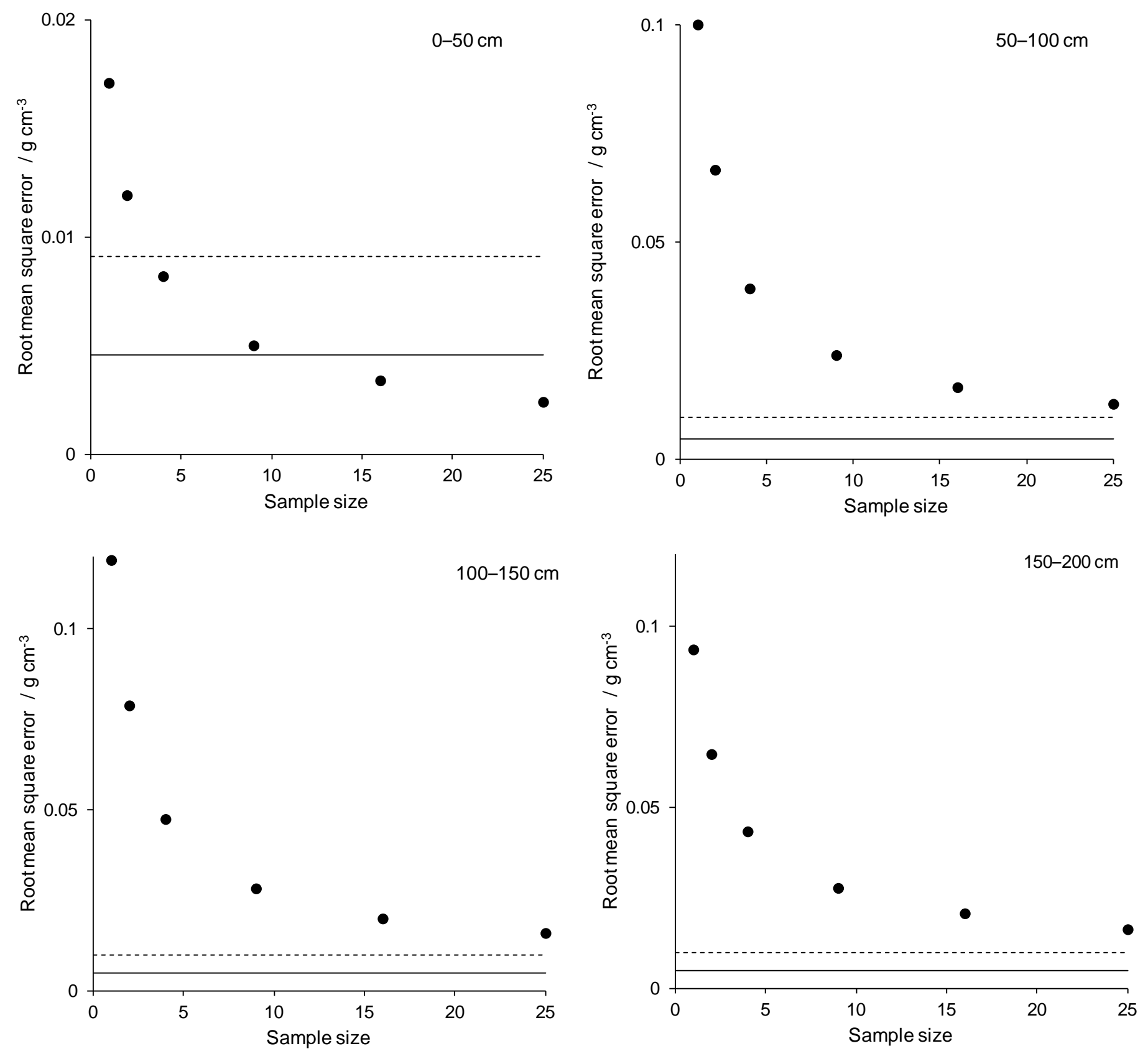

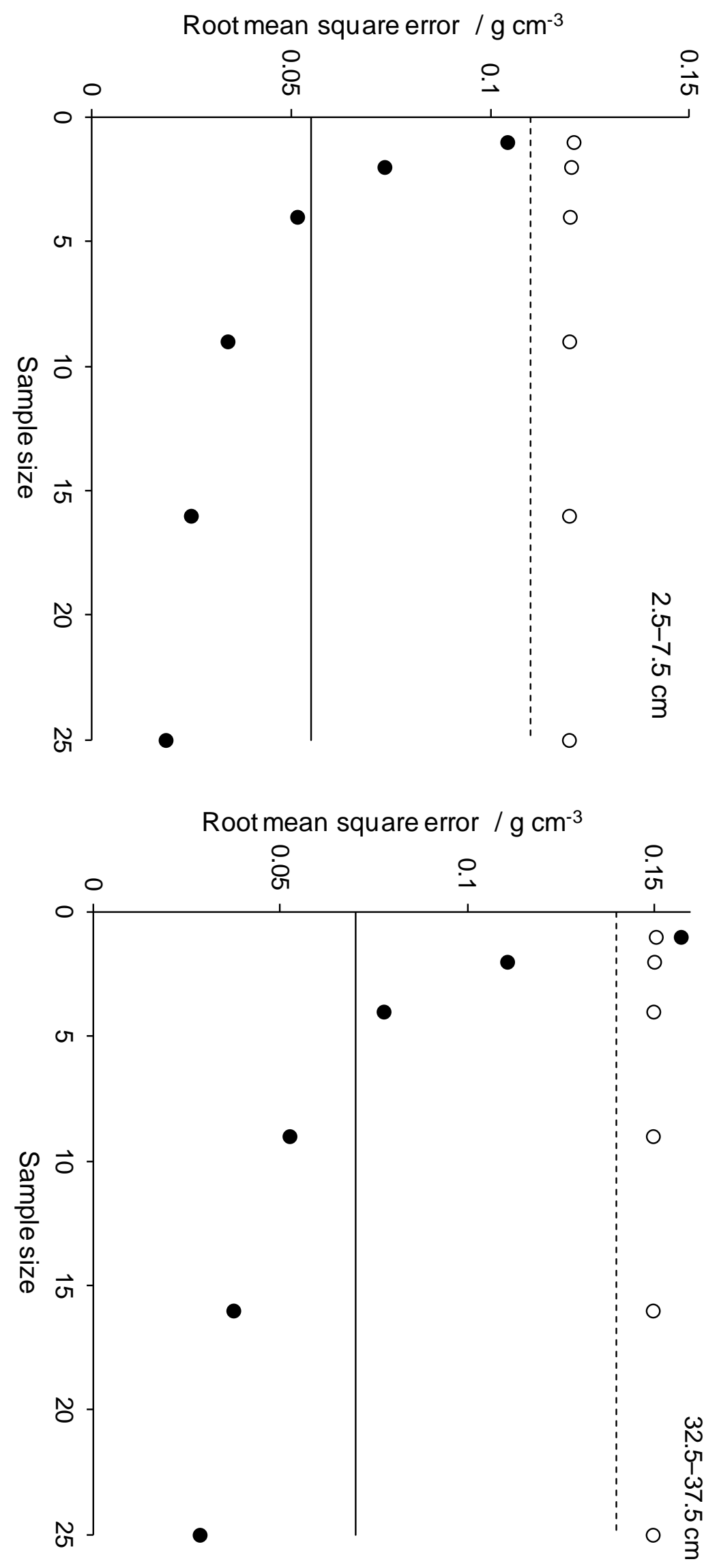


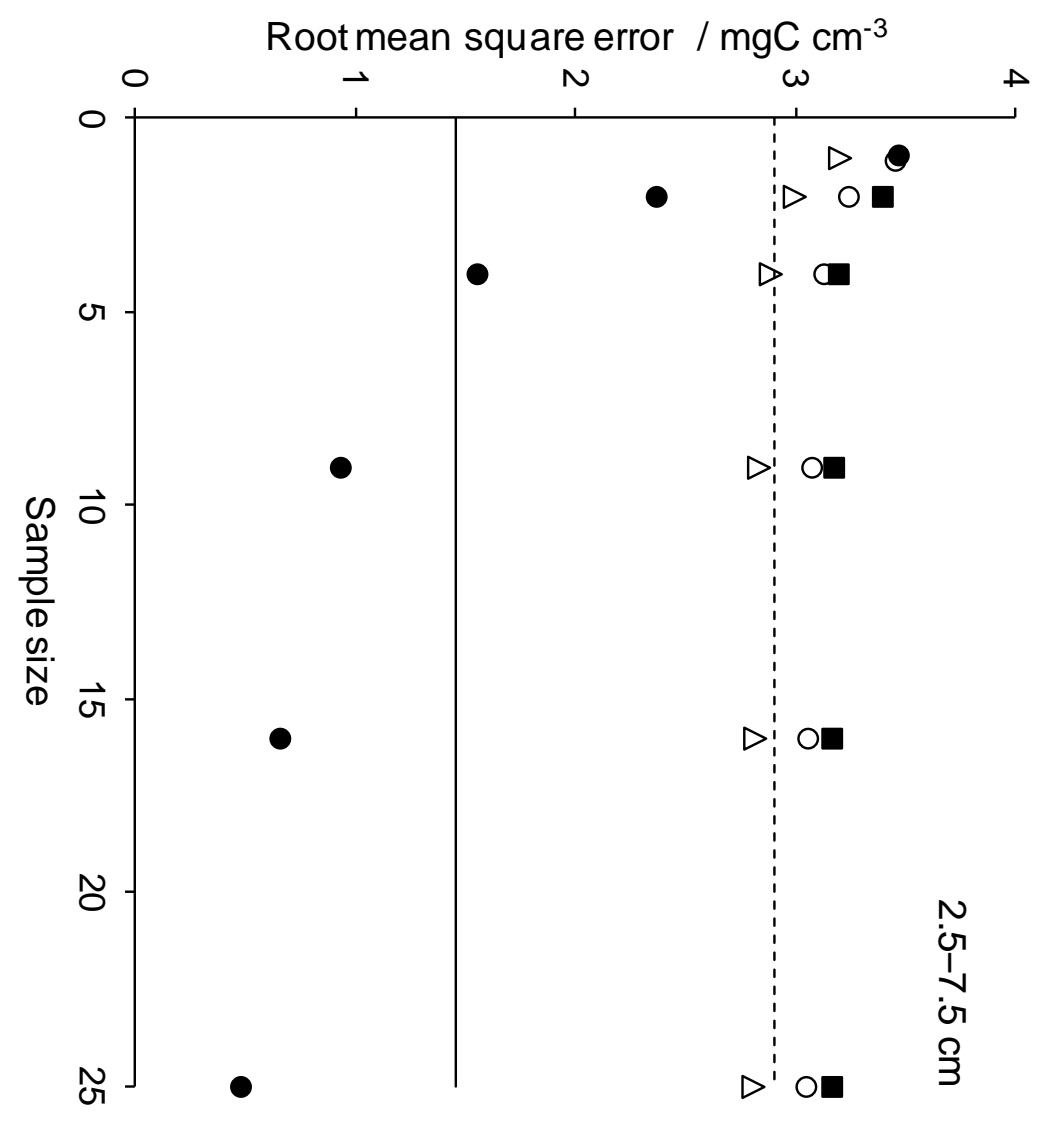

Root mean square error $/ \mathrm{mgC} \mathrm{cm}^{-3}$

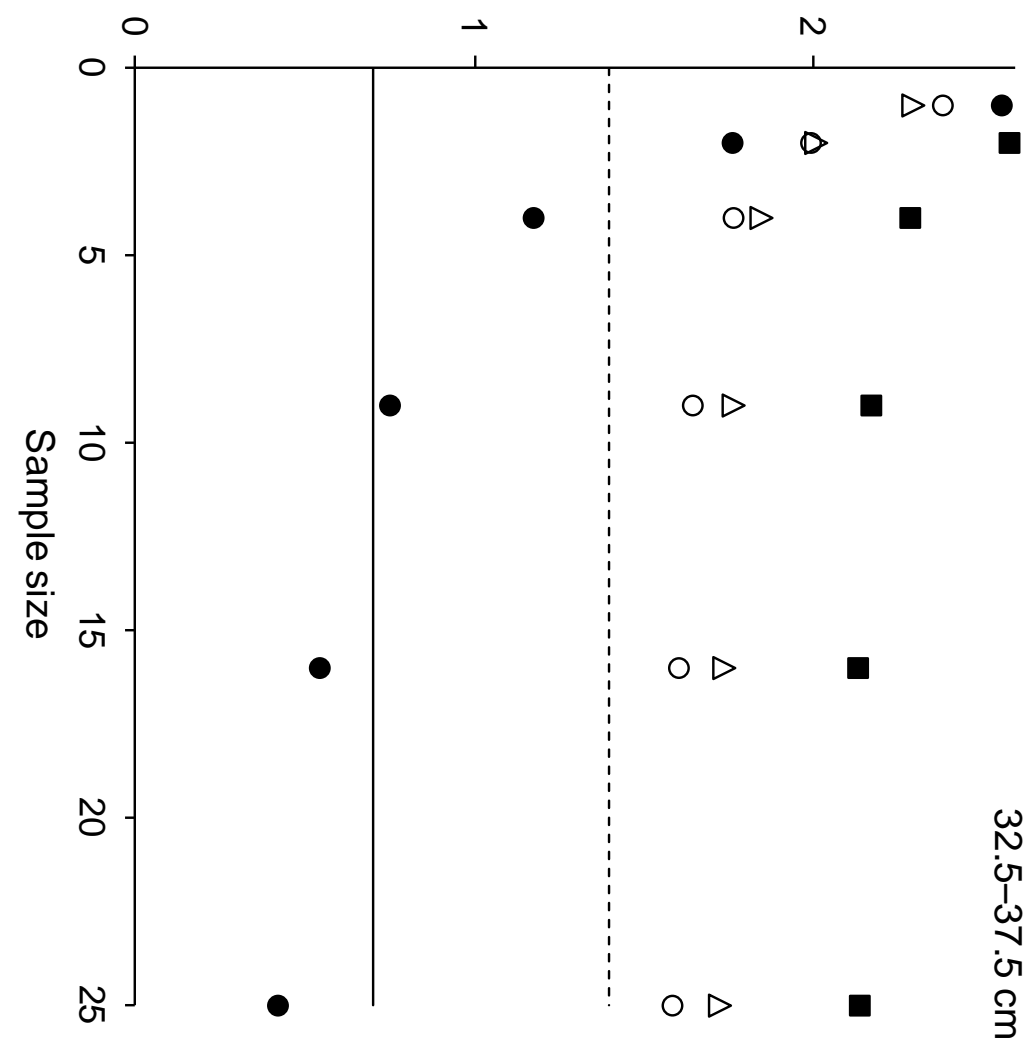

